\title{
HISTOIRES DE PIONNIERS FRANÇAIS DANS L'OUEST CANADIEN : LE CAS D'UN HÉROS MALGRÉ LUI DE MARCEL DURIEUX ${ }^{1}$
}

\author{
Denis LACROIX, Université de l'Alberta \\ Sathya RAO, Université de l'Alberta
}

\section{Panorama de la littérature des Français de l'Ouest et de l'Alberta}

Les années 1980 voient la publication de plusieurs ouvrages écrits par des pionniers français ayant vécu ou séjourné temporairement dans l'Ouest canadien. Au nombre de ces publications, on compte Les Français dans l'Ouest (1980) de Donatien Frémont, Dans la prairie canadiennelOn the Canadian Prairies (1982) de Gaston Giscard et d'Un héros malgré lui (1986) de Marcel Durieux. Suivront Conquérants du sol (1986) de Jules Lamy, Soupe maigre et tasse de thé (1994) de Christine de La Salmonière et Les détours du destin (2001) de Jean Bâcle. À l'exception du récit de Gaston Giscard publié par le Canadian Plains Research Centre, les autres ouvrages se trouvent tous sur le catalogue des Éditions des Plaines qui se font une spécialité de ce type de littérature. À ces ouvrages publiés au Canada, s'en ajoute une poignée d'autres parus quelques décennies auparavant en France comme La Terre pour rien (1907) de Jean du Saguenay et L'aisance qui vient de Louis et Jean ${ }^{2}$ (1911) publiés tous les deux aux éditions Bloud connues pour leur orientation catholique, La Pointe-aux-Rats (1907) et Dans l'Ouest canadien (1915) de Georges Forestier, Au Pays des érables (1939) d'Amélie ConstantinBompard, Athabasca, terre de ma jeunesse (1972) de Pierre Maturié dont une traduction anglaise vient d'ailleurs de paraître aux Presses d'Athabasca ou encore $D u$ vent, Gatine: un rêve américain (1989) de Jacques Bertin. Il convient également de mentionner les quelques écrits de pionniers francophones à l'image de Croquis du far-west canadien (1928) et Le Robinson de la Red Deer (1930) du Suisse André Borel ainsi que Retour à Calgary (1985) du Belge Bernard Gheur. Outre le fait que ces textes portent tous sur les Prairies (en particulier l'Alberta et le Manitoba) et sont le fait d'auteurs français, ces ouvrages n'ont finalement que très peu de choses en commun. Certains, comme La Terre pour rien et La Pointe-aux-Rats, répondent à des motivations idéologiques à savoir favoriser l'immigration au Canada ${ }^{3}$ ou, au contraire, en dénoncer le mythe. D'autres, à l'image d'Athabasca, terre de ma jeunesse, affichent des prétentions littéraires (Cadrin). Une autre ligne de partage notable se dessine entre les textes à 
fort contenu autobiographique (par exemple, Soupe maigre et tasse de thé et Dans la prairie canadienne) et ceux composés à partir de sources secondaires (correspondances, archives, etc.) comme Retour à Calgary et Du vent, Gatine: un rêve américain. À la fois complexe et hétéroclite, ce corpus a fait l'objet de plusieurs études. On a pu ainsi faire valoir la diversité des points de vue des pionniers français sur l'entreprise de colonisation de l'Ouest (Eygun), insister sur la spécificité historique et littéraire du point de vue des pionnières (Pénisson, «Louise d'Hellencourt »; Harvey) dont le point de vue est généralement passé sous silence ou bien s'interroger sur le genre auquel appartient les écrits de pionniers (Mocquais). La somme de ces recherches a sans aucun doute contribué à faire entrer cette littérature dans le patrimoine littéraire et historique de la francophonie de l'Ouest canadien tandis que bon nombre de ces auteurs ont trouvé une place dans le Dictionnaire des artistes et des auteurs de l'Ouest canadien dirigé par Gamila Morcos et paru aux Presses de l’Université de Laval.

De tous les Français ayant écrit sur l'Ouest, ce sont sans aucun doute Georges Bugnet et Maurice Constantin-Weyer qui ont suscité le plus grand intérêt critique. En plus des nombreux travaux consacrés à leurs œuvres ${ }^{4}$, ces deux écrivains ont fait chacun l'objet d'un numéro spécial (volume 11, nos 1 et 2) des Cahiers Franco-canadiens de l'Ouest intitulé «Visages de Georges Bugnet ». La confrontation entre Bugnet et Constantin-Weyer permet de mesurer l'écart profond qui sépare deux visions de l'Ouest formulées par des écrivains français ${ }^{5}$. Dans son ouvrage Les Français dans l'Ouest canadien, Frémont dresse des portraits pour le moins contrastés de l'auteur de La Forêt et de celui de Vers l'Ouest : tandis que le premier s'est « révélé être un observateur pénétrant de la nature et de l'Ouest canadien » (196), le second «se classe dans la catégorie des amateurs et des fantaisistes qui, quand il aborde l'histoire du pays, la romance au point de la défigurer totalement; mais comme peintre de la nature on peut l'admirer sans réserve » (75). Alors que Constantin-Weyer doit faire face à la fois à la critique canadienne (et en particulier québécoise) qui l'accuse d'avoir caricaturé les Français-Canadiens tout autant qu'aux reproches de son compatriote Frémont ${ }^{6}$, Bugnet, quant à lui, est loué en raison de son intégration exemplaire au milieu albertain. C'est d'abord et avant tout par son attachement indéfectible à une identité canadienne, qu'il s'efforcera de découvrir notamment à travers son œuvre romanesque, que Bugnet se distingue de ses compatriotes. Des articles comme «Canadiana », « Montaigne et les Canadiens » et « Pour 'l'esprit canadien' » regroupés dans l'anthologie intitulée Albertaines témoignent du désir de Bugnet de prendre distance avec la France et ce qu'il appelle « les modes 
de Paris » (158) dans le but de se construire une identité proprement canadienne : «Comment parviendrons-nous jamais à être canadien, à être nous-mêmes, quand nous travaillons sans répit pour ressembler à d'autres »(125). L'auteur trouvera une expression sublimée de cette identité dans une mystique de la nature qu'il développera en particulier dans Nypsia. À l'inverse, Constantin-Weyer excellera à donner une description plus vivante et sensuelle de la nature où l'érotique des paysages grandioses prendra le pas sur la recherche d'une communion mystique avec la nature :

Bugnet n'a pas la sensibilité raffinée de Constantin-Weyer, c'est pourquoi ses descriptions n'en ont ni la couleur, ni le mouvement ni la passion. Il ne ressent pas, il perçoit.... Et puisqu'elles découlent d'une expérience mystique, ses descriptions de la Nature sont en quelque sorte transcendantales : elles dépassent le domaine des connaissances pour sonder l'absolu. (Farquhar 142)

En 1984, l'universitaire d'origine belge Paulette Collet est l'une des premières à rompre le silence académique qui pèse sur les auteurs français ayant écrit sur l'Ouest en proposant une anthologie intitulée Les Romanciers français et le Canada (1842-1981). Elle y inclut des textes d'écrivains comme Forestier et Bugnet. Dans sa préface, l'universitaire explique clairement l'objectif qui guide son anthologie :

Sans les romanciers français, les Prairies feraient figure de parent pauvre dans la littérature francophone du Canada, la plupart des écrivains étant originaires du Québec et préférant parler de ce qu'ils connaissent à fond.... Mais, somme toute, malgré l'activité littéraire qui se manifeste depuis quelques années dans les milieux francophones des Prairies, celui-ci serait assez mal représenté dans le roman de langue française s'il n'y avait eu Constantin-Weyer, Maurice Genevoix, Georges Bugnet et des auteurs plus obscurs comme, par exemple, Georges Forestier, paysagiste de talent. (10)

L'autre point sur lequel insiste Collet, est la valeur historique et sociologique de cette littérature qui renseigne sur l'immigration française du début du vingtième siècle. Dans le sillage de son anthologie, la critique fait paraître une étude qui regroupe une série de vignettes biographiques présentant les principaux romanciers français de l'Ouest et du Nord-Ouest canadiens. L'un des intérêts de cette étude est qu'elle met en lumière la diversité des parcours biographiques et littéraires de ces écrivains français dont certains, comme Bugnet, ont élu domicile dans les Prairies alors que d'autres, à l'instar de Forestier et de Constantin-Weyer, n'y ont fait que passer.

Un peu moins d'une quinzaine d'années plus tard, paraîtra le Dictionnaire des artistes et des auteurs francophones de l'Ouest canadien qui recense plus d'une vingtaine d'écrivains et de poètes contemporains nés en France ou d'origine française dont Jean d'Artigue, Jacqueline 
Barral, Roland Bonvalet, Jean-Antoine Bour, Monique Genuist et Jules Lamy pour n'en citer que quelques-uns. S’ils adoptent une perspective présentée comme «francophone », les éditeurs du dictionnaire n'éprouvent cependant pas la nécessité de faire état de la diversité linguistique et culturelle que ce terme recouvre ${ }^{7}$. Qu'en est-il de la représentation française dans le dictionnaire dans le détail ? Sur la vingtaine d'auteurs français recensés, plus des deux tiers sont décédés. Pour ce qui est des vivants, leur moyenne d'âge dépasse cinquante ans. Si la plupart d'entre eux étaient ou sont encore établis au Manitoba, environ un tiers a élu domicile temporairement ou définitivement en Alberta. Une caractéristique sociologique notable de ces auteurs français de l'Ouest est qu'ils sont, pour la grande majorité d'entre eux, universitaires. C'est notamment le cas d'Alexandre Amprimoz, de Jacqueline Barral, de Jean-Antoine Bour, de François-Xavier Eygun et de Monique Genuist. Nourries par une connaissance intime de l'histoire de la littérature, leurs œuvres tranchent tant par leur style que par leur contenu avec les récits autobiographiques de pionniers.

\section{Une littérature de pionniers? Le cas d'Un héros malgré lui de Marcel Durieux}

Dans une communication donnée lors du 4 e colloque du CEFCO en 1984, le canadianiste Pierre-Yves Mocquais s'attachait à comparer Un héros malgré lui/Ordinary Heroes de Marcel Durieux et Dans la prairie canadienne de Gaston Giscard. Son propos était de comprendre dans quelle mesure ces deux textes pouvaient être apparentés au genre littéraire de la chronique. Au terme de sa comparaison, le critique en arrivait à la conclusion selon laquelle «Ordinary Heroes de Marcel Durieux et Dans la prairie canadienne de Gaston Giscard ne sont pas des chroniques mais elles en ont la fonction » (123). La raison qu'invoque le critique est la suivante :

Au début du siècle, dans l'Ouest canadien, que ce soit dans l'absolu, ou qu'il s'agisse de la perception que les premiers pionniers eurent de leurs aventures, dans un cas comme dans l'autre, l'histoire ne fait que commencer. Or il s'agit là d'une histoire où le quotidien l'emporte sur le grand fait historique car le recul nécessaire à l'analyse des évènements, à l'évaluation de leur importance relative, ce recul n'existe pas. (123-124)

L'intérêt majeur de l'étude de Mocquais est d'interroger l'articulation complexe entre forme littéraire et temporalité. Dans cette mesure, cette étude va plus loin que l'interprétation que proposent Roger Motut et Maurice Legris dans leur préface. Pour ces derniers, Un héros malgré lui se limite à être le journal d'un colon ordinaire qui « quoique fermier et non homme de lettres, a écrit un journal qui est l'œuvre d'un homme sensible et intelligent » (Legris 3). La sincérité du 
jugement moral porté sur l'auteur - dont les préfaciers louent tour à tour la compassion, la loyauté et la religiosité - contrebalance la faiblesse de l'analyse littéraire qui tient en tout et pour tout dans l'énoncé suivant : «Profondément religieux, il savait exprimer très simplement et de façon concrète à cet égard » (3). Pour notre part, nous pousserons un cran plus loin l'hypothèse de Mocquais en considérant Un héros malgré lui comme un texte dont la fonction est celle non pas d'une chronique ${ }^{8}$ mais d'un roman. Par là, nous voulons dire que le récit de Durieux met consciemment en œuvre des stratégies de fictionnalisation qui témoignent d'un effort de distanciation à l'égard du quotidien narré contrairement à ce qu'affirme Mocquais. Plus exactement, nous montrerons que cette distanciation se manifeste de trois façons, soit : 1) l'incursion de la grande Histoire dans la chronique ordinaire du pionnier, celle-ci finissant par se subordonner à celle-là ; 2) la conscience linguistique exacerbée du narrateur qui s'attache à rendre compte dans le texte du multilinguisme qui caractérise la société pionnière ; 3) l'interpellation fréquente du lecteur par le narrateur qui acquiert ainsi une voix excédant le simple récit impersonnel (ou peut-être trop personnel au point d'en être monologique) de l'exposition des faits du quotidien. Nous considérons que c'est dans ce triple effet de distanciation historique, linguistique et narratif s'élabore l'ambition romanesque d'Un héros malgré lui.

\section{a) La question du temps}

Contrairement à ce qu'avance Mocquais, Un héros malgré lui ne se contente pas de narrer les événements au fur et à mesure qu'ils surviennent. Organisé certes selon une chronologie linéaire qui rend compte de la progression de Durieux et de sa famille vers l'Ouest, Un héros malgré lui présente cependant de nombreuses anachronies ${ }^{9}$. Certains événements, compte tenu de l'importance qu'ils revêtent aux yeux de l'auteur, font l'objet de plus amples développements dans le récit. C'est le cas notamment de la première expérience de travaux dans les champs au Manitoba, des rigueurs du premier hiver, des feux de prairie, de la mort de l'abbé Lainez et de la nuit de Noël. Rythmant le quotidien d'un grand nombre de colons, certains de ces événements - à l'image, par exemple, des feux de prairie - ont été utilisés par les éditeurs comme autant de têtes de chapitre permettant de structurer le récit de Durieux, initialement conçu comme « une narration continue » (Legris 6). 
Comme le note Mocquais, les prolepses constituent un des dispositifs narratifs majeurs du texte. Il est essentiel ici de rappeler qu'entre la situation énoncée et le moment de son énonciation a pris place un événement majeur, c'est-à-dire la Première Guerre mondiale dont la présence hante le récit de Durieux ${ }^{10}$. Cette présence subreptice qui donne lieu à plusieurs prolepses et commentaires à valeur proleptique sur lesquels nous reviendrons, renvoie à son tour à un autre événement historique qui, lui, relève du passé. Il s'agit en l'occurrence de la guerre franco-prussienne de 1870 durant laquelle le père de Marcel fut fait prisonnier (Durieux 61; 219). Cet épisode alimentera une certaine animosité à l'égard des Prussiens que l'on retrouve, par exemple, chez le Suisse Karl Stettler (94). Ce qui n'empêchera pas le père Durieux de se lier d'amitié avec un Allemand, M. Zimmerman, dont le narrateur prend bien soin de préciser qu'il « se fiche du Kaizer » (89) et qu'il rêve de visiter la France (90). Au final, c'est donc la solidarité entre colons qui semble l'emporter sur les vieilles rancœurs européennes.

Linéaire en apparence, le récit de Durieux est en fait plus complexe qu'il n'y paraît puisqu'il s'organise selon deux axes temporels. Le premier de ces axes pourrait être qualifié de transhistorique; il se caractérise par l'évocation dans le récit d'événements futurs liés à la Première Guerre mondiale. Ces événements interviennent sous la forme de prolepses ponctuelles qui opèrent la jonction entre le récit principal de l'installation (qui correspond au niveau de la diégèse) et cette «autre histoire » à venir (242) - celle de la Grande Guerre - sur laquelle se conclut Un héros malgré lui. Pourtant consécutives l'une de l'autre, ces deux histoires se télescopent non seulement dans la narration, mais au niveau de sa poétique même. Ainsi le narrateur évoque-t-il souvent ses souvenirs de guerre dans un contexte bien particulier, à savoir celui du combat contre les phénomènes naturels (en l'occurrence, la tempête et le gel) qui est le lot des colons :

Je fus réveillé brusquement par un violent soubresaut de la toile de la tente; la tempête était complètement déchaînée, je n'eus même pas le temps de penser à la sinistre prophétie du parent de l'ami Musy au Sault-au-Récollet : «vot maison... enlevée comme eune bouète d'allumettes », qu'un craquement allant en s'amplifiant (le premier obus que j'entendis en 1914 me rappela ce déchirement) s'acheva en un fracas de coup de tonnerre. (123)

Jean partit, mais il ne revint pas ! Non pas qu'il périt gelé cette nuit-là, non ! Rassurez-vous, mais son destin l'appelait à une fin plus glorieuse. Son échéance était sur le versant du Mort-Homme à Verdun dans la nuit du 30 avril au $1^{\mathrm{er}}$ mai 1916.... (199) 
En d'autres termes, la rude réalité du combat contre la nature constitue en quelque sorte le point de suture entre l'expérience présente du colon et celle à venir du soldat qu'a été, tour à tour, Durieux. L'emploi de termes et de locutions comme «rappeler à l'ordre », « offensive », «mouvement tournant», « coup de Jarnac», «contre-offensive » et «bataille » empruntés au champ sémantique de la guerre, témoignent de la parenté entre la lutte contre les éléments naturels et l'expérience de la guerre.

C’était réellement féérique, cette illumination aérienne; on serait resté des heures en contemplation, mais le froid était là qui nous rappelait à l'ordre [nous soulignons] avec la nuit, le «chinook » avait été absorbé par la condensation. Les jours suivants, l'hiver sembla déclencher une nouvelle offensive [nous soulignons]. Les génies malfaisants, Maîtres des étendues glacées de l'Alaska, parurent furieux d'avoir été surpris par le mouvement tournant [nous soulignons] du «chinook ». Mais ce coup de Jarnac [nous soulignons] qui les avait momentanément bafoués fut durement châtié.... (161)

À ce moment, une série de tempêtes épouvantables marqua une contreoffensive de l'hiver [nous soulignons].... (214)

La neige avait commencé de tomber et le vent se levait rapidement. À trois heures, bien qu'il commençât à faire nuit, nous continuâmes jusqu'à l'endroit près des Bailleux où, cinq ans plus tôt, nous avions dû traverser, retraverser et tourner en rond. Cinq ans... et c'était toujours la même vie rude; la même bataille [nous soulignons] pour la survie. (233)

Le conflit entre les temporalités - à savoir le présent simple du quotidien et le futur proche de la grande Histoire - situe bien la position ambigüe de nombre de colons français qui ont dû hypothéquer leur rêve d'une vie meilleure au nom de la défense de leur Patrie. Ce fut le cas de Durieux qui, victime d'une intoxication au gaz empoisonné dans les tranchées, n'a plus été en mesure de retourner au Canada. En définitive, la France n'a jamais cessé d'être présente dans le récit du jeune pionnier. La présence de cette double temporalité va jusqu'à faire peser un doute sur les circonstances qui ont fait ou feront du narrateur un «héros malgré lui », martyr de la grande Histoire : s'agit-il de la colonisation ou bien de la guerre ? Non sans une certaine ironie, le récit de Marcel Durieux se conclut sur la mobilisation à venir du jeune Fournier :

Le malheureux Fournier ne prendrait pas Claire Randon pour femme. Deux mois plus tard, il aurait un fusil Lebel dans les mains... et il serait le premier du contingent français de Lowden Lake appelé aux armes à tomber sur le champ de bataille, le 18 septembre au Chemin des Dames à la fin de la bataille de la Marne. Mais ça, c'est une autre histoire. (242) 
Comme nous l'avons suggéré, il n'est pas certain que l'histoire de la Grande Guerre soit si étrangère au récit de Durieux dont elle constitue en quelque sorte l'horizon funeste (son point d'arrimage à la grande Histoire, pourrait-on dire) qui pointe au fil du texte pour finir par faire irruption dans la diégèse et l'interrompre. En définitive, la guerre hante Un héros malgré lui en ce sens où elle constitue à la fois la clôture du récit qui signale la rupture avec le Canada et l'analogue du combat que livre le colon contre la nature.

L'axe transhistorique que nous venons de caractériser se trouve combiné à un autre axe temporel qui concerne plus spécifiquement l'économie même de la diégèse. Celui-ci donnera lieu à plusieurs analepses et prolepses internes sur lesquelles nous reviendrons. Il est significatif de constater que les premières sont proportionnellement beaucoup moins nombreuses que les secondes, ce qui en dit long à la fois sur l'absence de nostalgie ${ }^{11}$ de Durieux qui prétend faire de son travail un échappatoire, et l'importance de la Première Guerre mondiale qui constitue un repère (extra-)référentiel majeur du récit. Parmi les prolepses internes les plus notables, on compte la malédiction lancée par l'ami de la famille Charles Musy (46), laquelle hantera un certain temps Durieux (123) sans pour autant compromettre la marche de la famille vers l'Ouest. Quant au décès de la mère du héros, elle est annoncée par une série d'événements tragiques initiés par la mort de l'abbé Laisnez:

Au moment de quitter la maison mortuaire, je me retournai instinctivement vers ce coin de la première sépulture de toute la région et je sentis aussitôt ma poitrine se serrer douloureusement, car je venais de voir, en même temps que le mien, le regard de ma mère se poser avec une sorte d'envie sur la dépression de terrain où reposait, maintenant, son modèle en courage et résignation. (196)

Ainsi, la mort de l'abbé Laisnez des suites d'une maladie chronique est suivie d'un enchaînement de mauvaises nouvelles (décès du Dr. Authenec, tempêtes «épouvantables », amputation des deux annulaires de Boulot) qui culminent avec la mobilisation. On notera également la présence de quelques autres prolepses apportant, quant à elles, simplement des éléments d'information (26; 61) sans incidence majeure sur l'action. Pour ce qui est des analepses, la plus représentative d'entre elles se situe en ouverture du chapitre VIII ; elle vise essentiellement à rappeler un événement qui a été omis dans le fil de la narration, assurant par là même une fonction complétive :

J'ai voulu anticiper en racontant, tout d'une traite, la pénible fin de l'abbé Laisnez ; aussi, nous reviendrons un peu en arrière, à la Noël de l'année 
précédente, cette mémorable nuit où nous avons bien failli laisser nos corps se geler parmi les collines de l'ouest de Lowden Lake. (197)

\section{b) Hétéroglossie des accents et métalepses}

Une autre caractéristique majeure du récit de Durieux, qui aura échappé aussi bien à l'attention des préfaciers qu'à celle de Mocquais, est sa conscience exacerbée des accents tant régionaux que nationaux dont l'auteur ira jusqu'à transcrire certaines des spécificités phonostylistiques $^{12}$. On ne compte en effet plus ces accents et patois représentés, lesquels manifestent l'extrême diversité linguistique et culturelle des sociétés pionnières des Prairies : canadiens-français (32), allemand (88), bruxellois (92), béarnais (110) ou encore roubaisien (171). Dans certains cas, il s'agit de parlers très spécifiques à l'instar de ce jargon dans lequel s'exprimait le plus âgé des Laisnez «composé de patois de la frontière franco-belge, comme s'expriment à peu près les ouvriers de Mouscron... » (177). Durieux est d'autant plus en mesure d'identifier ce dialecte qu'il est lui-même originaire du Hainault et semble connaître le «patois des mineurs du Nord de la France » (152). Le narrateur n'hésite pas non plus à marquer les variations diastratiques (en mobilisant aussi bien les ressources lexico-sémantiques que la prononciation) pour particulariser davantage le profil sociolinguistique des locuteurs. Ainsi les répliques rapportées du conte de Bouville (174) et surtout de l'un des frères Laisnez (178) se distinguent-elles par leur niveau de langue élevé et leur complexité syntaxique. Ce désir d'authenticité pousse l'auteur à faire parler certains locuteurs dans leur anglais d'origine (30, $126,127,142,148)$ et à proposer presque systématiquement à la suite une traduction entre parenthèses. Cela dit, il convient de noter que l'anglais cède souvent la place tantôt au français tantôt à un français anglicisé lorsque les répliques sont trop longues $(127 ; 140)$, et ce donc au mépris du souci affiché de réalisme. Fidèles dans leur ensemble, les traductions ont tendance à privilégier davantage le message que la forme comme en témoigne la non-traduction des marqueurs pragmatiques, interjections et, le cas échéant, l'emploi de l'étoffement - en procédant, par exemple, à l'ajout de ressources lexicales - à des fins de clarification ${ }^{13}$ :

- Say Frenchie? I believe you laughed at me, didn't you ?

- No, no ! Dis-je en reprenant mon calme, I am laughing to prevent crying, you see ? (je ris pour ne pas pleurer.)

- Oh ! Well, I mean to measure up the spoiled parts of it. (On va voir le bois qui manque, en mesurer les pièces endommagées). (126) 
L'hétérolinguisme (Grutman) dont il est ici question est une construction linguistico-narrative (combinant la retranscription du phonétisme des accents et l'utilisation de la forme dialoguée) qui rend compte de la réalité historique de la société pionnière de l'Ouest où cohabitaient des hommes issus de diverses nationalités ${ }^{14}$ et situations sociales. C'est peut-être la chorale, formée à l'occasion de l'inauguration de la nouvelle église, qui met le mieux en scène la réalité multiculturelle de l'Ouest :

Quant à la chorale, ce fut un raccourci du monde catholique de l'Alberta centrale; en chantant du latin, cela se fondait à peu près; en anglais, cela grinçait un peu, mais dans les cantiques français, c'était quelque chose d'inénarrable ! Je mentionnerai seulement les principales origines des chanteurs : d'abord les Français, Belges et Suisses ; les Canadiens français et les Métis, les Irlandais, Anglais et Américains, et enfin les Tchèques, Hongrois, Polonais ! À l'exécution, j'avais dans mon oreille droite la voix retentissante de Swoboda de Pilsen, employé de banque, qui chuintait toutes les consonnes, et dans mon oreille de gauche, un Métis, neveu de Xavier de Content, qui grasseyait à pleine bouche. (210)

Le concept de polyphonie ${ }^{15}$ s'avère parfaitement approprié compte tenu des circonstances pour rendre compte du caractère multiculturel de la société dans laquelle évolue Durieux. Extrêmement hétéroclite, cette société se fédère cependant autour de l'attachement au catholicisme et du sentiment de solidarité qui naît du combat commun contre la nature. Le narrateur n'évacue pas pour autant les préjugés touchant les us et coutumes des diverses communautés nationales y compris les Français :

Pour une fois, Marcel, je voudrais pas faire honte à la France, mais je dois dire que presque tous les Français du Centre que je connais ici, hé ben, ça n'est pas le modèle de la grande propreté. C'est de même chez certains Irlandais ou même des Américains de basse classe. Les modèles, c'est les Danois et les Suédois ; mais si vous voulez trouver le plus grand triomphe de la plus grande cochonnerie, tu n'as qu'à aller voir chez quelqu'un qu'on vous dira Juif Russe. $(85)$

Notable dans les dialogues, l'hétéroglossie touche également le corps de la narration qui est truffée d'emprunts à l'anglais. Loin d'être cosmétiques, ces emprunts, qui servent souvent à nommer une réalité spécifique du pays d'accueil, sont accompagnés de traductions : “'chaps' (pantalons en gros cuir et fourrure) » (Durieux 112), «Buffalo grass, l'herbe aux buffles » (114), « shingles (bardeaux de cèdre qui servent d'ardoises)»(126), «green horn (bleu apprenti) » (121), « baking powder (levure alsacienne) » (159). On retrouve un phénomène similaire dans les dialogues $(127 ; 147)$. L'emploi de termes étrangers ajoute à l'authenticité du récit en même 
temps qu'il lui confère un caractère informatif voire pédagogique que renforcent les traductions à valeur explicative. Témoignant du degré d'intégration du narrateur à sa nouvelle réalité culturelle et linguistique, ces emprunts d'ordre strictement lexical «métissent » dans une certaine mesure son français sans que cela n'atteigne cependant les proportions du français anglicisé parlé par le Métis. C'est probablement ce même souci pédagogique qui poussera l'auteur à faire quelques digressions afin d'expliquer, par exemple, la réalité juridique qui se rattache aux notions de « terre » et de « terrain » $(75)$.

Une dernière caractéristique du récit de Durieux qui le rapproche du roman (notamment parodique) plutôt que de la chronique est la multiplication de métalepses (Genette) comme en témoigne la liste d'exemples suivants : «[...] vous avez certainement déjà vu un château de cartes gauchir tout à coup » (124), «[...] et savez-vous de combien de degrés il dégringola au bout de dix minutes » (136), «[...] vous jugez si on courut le plus vite possible [...]» (156), «[...] devinez qui nous rattrape dans les collines $[\ldots] »(163), «(\ldots)$ vous vous souvenez sans doute que $[\ldots] »(182), \ll[\ldots]$ alors vous comprenez facilement que $[\ldots] »(185)$. Le narrataire se trouve ainsi régulièrement interpellé, ce qui contribue créer un lien de proximité avec le narrateur; lien que ce dernier peut exploiter pour accentuer ses effets ou bien mobiliser l'attention du lecteur.

\section{Conclusion}

Y a-t-il une littérature française de l'Ouest canadien ? Il est difficile de répondre par l'affirmative compte tenu de la diversité de textes, d'auteurs et de postures énonciatives. Faut-il l'intégrer au patrimoine littéraire franco-albertain ou plus généralement canadien, la traiter comme une littérature migrante ou bien encore la rapatrier dans le giron de la littérature française nationale (par exemple, sous l'étiquette exotique du roman d'aventures). Il ne fait aucun doute que cette incertitude identitaire frappe également ceux qui en sont les auteurs. À cet égard, la divergence entre les postures de Georges Bugnet et de Michel Constantin-Weyer est emblématique. Dans le cadre de cet article, notre propos a été moins de chercher à situer une bonne fois pour toutes cette littérature qu'à la problématiser au sens où le préconisait Gilles Deleuze. En d'autres termes, la littérature française de l'Ouest existe précisément par la somme de questions et de résistances (institutionnelles, littéraires, idéologiques, etc.) que soulève le principe même de son existence (y compris même la prétention qu'elle a à se constituer en 
littérature). Appartenant à ce corpus problématique de la littérature française de l'Ouest, le récit de Durieux a d'abord été traité, non sans condescendance, comme un témoignage historique pour ensuite se voir hisser au statut de chronique. Pour notre part, nous avons formulé l'hypothèse qu'Un héros malgré lui fonctionnait plutôt comme un roman compte tenu : 1) de son mode complexe de construction de la temporalité qui le rattache malgré tout à la grande Histoire contrairement à ce qu'avance Mocquais ; 2) de son aspect relativement polyphonique et hétéroglossique (qui témoigne en soi d'une certaine surconscience linguistique de l'auteurnarrateur), et enfin ; 3) de la présence fréquente de métalepses qui présupposent l'adresse à un lecteur-narrataire, ce qui est plutôt étonnant pour un journal. L'intérêt d'une telle hypothèse est double : en premier lieu, elle oblige à une lecture d'autant plus attentive du récit qu'il se trouve élevé à la dignité de roman; en second lieu, elle ouvre des pistes inédites permettant de problématiser d'un point de vue littéraire l'expérience des pionniers (français). La plus productive d'entre elles réside, nous semble-t-il, dans la mise en mots proprement romanesques de l'expérience de la diversité culturelle et sociale qui la caractérisait.

\section{Sources primaires citées}

Bâcle, Jean. Les détours du destin. Saint-Boniface : Plaines, 2001.

Bertin, Jacques. Du vent, Gatine ! : un rêve américain. Paris : Arléa, 1989.

Bugnet, Georges. Albertaines : anthologie d'œeuvres courtes en prose. Saint-Boniface : Plaines, 1990.

Constantin-Bompard, Amélie. Au Pays des érables. Tours : Maison Mame, 1934.

Durieux, Marcel. Un héros malgré lui. Saint-Boniface : Plaines, 1986.

Forestier, Georges. La Pointe-aux-Rats. Paris : Plon, 1907.

---. Dans l'Ouest canadien. Paris : Plon, 1907.

Giscard, Gaston. Dans la prairie canadienne / On the Canadian Prairies. Regina : Canadian Plains Research Centre, 1982.

La Salmonière, Christine de. Soupe maigre et tasse de thé. Saint-Boniface : Plaines, 1994.

Lamy, Jules. «Conquérants du sol ». Dans la Terre promise. Saint-Boniface : Plaines, 1986.

Maturié, Pierre. Athabasca, terre de ma jeunesse. Paris : La Pensée Universelle, 1972.

Saguenay, Jean du [Léopold Léau]. Terre pour rien. Paris : Bloud, 1907. 
Viel, Louis et Jean du Saguenay [Léopold Léau]. L'aisance qui vient. Paris : Bloud, 1911.

\section{Bibliographie}

Cadrin, Gilles. «Pierre Maturié : un regard nostalgique sur la terre de sa jeunesse ». Actes $d u$ vingtième colloque du CEFCO 15-18 octobre 2003 : L'Ouest : directions, dimensions et destinations. Winnipeg : PU de Saint-Boniface, 2005. 407-420.

Collet, Paulette. Les Romanciers français et le Canada, 1842-1981 : anthologie. Sherbrooke : Naaman, 1984.

Durieux, Marcel. Un héros malgré lui. Saint-Boniface : Plaines, 1986.

Eygun, Francois-Xavier. «Divergences et convergences à travers deux romans : La Pointe aux rats (1907) et L'aisance qui vient (1911)». Actes du vingtième colloque du CEFCO 1518 octobre 2003 : L'Ouest : directions, dimensions et destinations. Winnipeg : PU de Saint-Boniface, 2005. 435-446.

Harvey, Carol. J. «Souvenirs réels ou romancés ? Les écrits de Christine de La Salmonière et d'Amélie Constantin-Bompard ». Actes du vingtième colloque du CEFCO 15-18 octobre 2003 : L'Ouest : directions, dimensions et destinations. Winnipeg: PU de SaintBoniface, 2005. 447-461.

Farquhar, Simone Paula. Anthe, ou, L'Ouest canadien dans l'œuvre de Maurice Constantin-

Weyer et de Georges Bugnet. MA thesis U of British Columbia, 1966.

Frémont, Donatien. Les Français dans l'Ouest canadien. Saint-Boniface : Blé, 1980.

---. Sur le ranch de Constantin-Weyer. Winnipeg : Liberté, 1932.

Genette, Gérard. Figures III. Paris : Seuil, 1972.

Grutman, Rainier. Des langues qui résonnent. L’hétérolinguisme au XIXe siècle québécois. Montréal : Fides, 1997.

Legris, Maurice et Roger Motut. Présentation. Un héros malgré lui. Marcel Durieux. Saint Boniface : Plaines, 1986.

Mocquais, Pierre-Yves. « Gaston Giscard et Marcel Durieux, chroniqueurs ? » Actes du

Quatrième Colloque du Centre d'études franco-canadiennes de l'Ouest tenu au Collège universitaire de Saint-Boniface les 23 et 24 novembre 1984 : La langue, la culture et la société des francophones de l'Ouest. Ed. Annette Saint-Pierre et Liliane Rodriguez. Saint-Boniface: Le Centre, 1985. 117-126. 
Morcos, Gamila. Dictionnaire des artistes et des auteurs francophones de l'Ouest canadien.

Sainte-Foy : PU Laval, 1998.

Motut, Roger. Maurice Constantin-Weyer : écrivain de l'Ouest et du Grand Nord. SaintBoniface : Plaines, 1982.

Papen, Jean. Georges Bugnet, homme de lettre canadien. Saint-Boniface : Plaines, 1985.

Pénisson, Bernard. «Louise d'Hellencourt et Christine de La Salmonière : deux Françaises au Manitoba ». Cahiers franco-canadiens de l'Ouest 12.2 (2000) : 153-179.

\section{Notes}

${ }^{1}$ Cet article est la version remaniée d'une communication présentée conjointement avec Denis Lacroix au colloque international «Mémoire, diasporas et formes du roman francophone contemporain » organisé par l'Université de Waterloo à Kitchener (Ontario, Canada) le 30 avril 2011.

${ }^{2}$ Les auteurs de l'ouvrage sont en fait Louis Viel et Jean du Saguenay [Léopold Léau]. Ce dernier était directeur de la Collection canadienne aux éditions Bloud.

${ }^{3}$ L'anticléricalisme qui sévissait en France au début du XXe siècle a favorisé l'immigration de catholiques français dans l'Ouest canadien. Les ouvrages de Jean du Saguenay destinés aux futurs colons avaient pour vocation d'aider à l'installation matérielle de cette immigration en leur prodiguant des conseils pratiques, par exemple, sur l'agriculture locale, les modalités d'attribution des terres ou encore les bons placements financiers.

${ }^{4}$ Compte tenu de l'importance de son œuvre, Maurice Constantin-Weyer a fait l'objet de nombreuses études (cf. la bibliographie, datant quelque peu, compilée par Roger Motut à la fin de son ouvrage Constantin-Weyer, écrivain de l'Ouest et du Grand Nord (1982). Ayant connu une reconnaissance littéraire se limitant aux frontières du Canada, Georges Bugnet a, pour sa part, fait l'objet d'une monographie intitulée Georges Bugnet, homme de lettres canadien (1985) dont l'auteur est Jean Papen.

5 À notre connaissance, la seule étude qui s'attache à comparer de façon systématique l'œuvre de Maurice Constantin-Weyer et celle de Georges Bugnet est le mémoire de maîtrise de Simone Farquhar dont le jugement est cependant fortement influencé par l'opinion négative que se fait Donatien Frémont de Vers l'Ouest.

${ }^{6}$ Dans l'essai intitulé Sur le ranch de Constantin-Weyer (1932), Donatien Frémont aura l'occasion de revenir en détail sur le séjour de Maurice Constantin-Weyer à Saint-Claude dans le Manitoba, ses mœurs douteuses ainsi que sur les erreurs historiques que contiennent ses romans.

${ }^{7}$ Les tableaux proposés dans l'introduction du Dictionnaire mettent davantage l'accent sur le dénombrement des minorités francophones ainsi que leur taux d'assimilation. De plus, il n'y est fait qu'une brève mention de l'immigration européenne. Il convient également de remarquer que le terme «auteur » est pris dans un sens très large puisqu'il inclut aussi bien les écrivains, les artistes visuels, les professionnels des médias, les critiques que les essayistes.

${ }^{8}$ Pierre-Yves Mocquais considère plus précisément Un héros malgré lui comme un journal dans lequel l'auteur se serait contenté de consigner les faits de la vie ordinaire tels qu'ils surviennent, suivant en cela l'interprétation de Legris et Motut.

${ }^{9}$ Nous empruntons ce concept ainsi que ceux de «prolepses » et d' " analepses » à l'approche narratologique de Gérard Genette (1972).

${ }^{10} \mathrm{Ce}$ point fondamental met en question la lecture de Pierre-Yves Mocquais puisqu'à travers l'événement en filigrane de la Première Guerre mondiale s'opère la jonction entre la grande Histoire et le quotidien du colon. C'est précisément dans cet espace liminaire que se tient l'auteur-narrateur Marcel Durieux.

${ }^{11}$ Suite au décès de ses parents, Marcel Durieux confie que « [1]e rythme immuable des saisons avec leurs tâches spéciales nous fit oublier notre chagrin » (228).

${ }^{12}$ Par exemple, le narrateur fera chuter le $l$ du pronom personnel masculin $i l$ dans les énoncés en français québécois : «...malheureusement dimanche i marche point....»(32).

${ }_{13}$ À ce titre, l'on peut dire que les traductions remplissent plutôt une fonction explicative voire pédagogique.

${ }^{14} \mathrm{Au}$ fil du récit de Marcel Durieux, on rencontre des Français, des Belges, des Écossais, des Britanniques, des Allemands, des Suisses, des Norvégiens, des Finlandais, des Chinois et des Polonais. 
${ }^{15}$ Ce concept est pris ici dans un sens beaucoup plus général que celui qu'il revêt dans le contexte bakhtinien. Le récit de Marcel Durieux est loin d'avoir la complexité narrative des œuvres de Dostoïevski. Cela dit, la volonté du narrateur de rendre compte de la diversité des accents fait en sorte que le récit fonctionne comme un roman à défaut d'en être véritablement un. 